\title{
Caracterización anatómica de cinco especies maderables provenientes de Madre de Dios y Ucayali, Perú
}

\author{
Anatomical characterization of five timber species from Madre de Dios and \\ Ucayali, Peru
}

Zarela Cerdán Obregón ${ }^{1, *}$ y Manuel Chavesta Custodio ${ }^{2}$

\begin{abstract}
Resumen
El objetivo del trabajo fue describir la estructura anatómica de la madera de las especies Aspidosperma excelsum Benth., Licania micrantha Miq., Buchenavia amazonia Alwan \& Stace, Laetia procera (Poepp.) Eichler y Sloanea laurifolia (Benth.) Benth. Las tres primeras fueron colectadas en Madre de Dios y las restantes en Ucayali, Perú. La descripción anatómica se realizó conforme a las normas de IAWA (1989) e IBAMA (1991); incluye información cualitativa y cuantitativa del xilema, presentándose características comunes como grano entrecruzado, porosidad difusa, platinas de perforación simples, punteaduras intervasculares alternas y apéndices en los elementos vasculares. Algunas características distintivas según especie fueron parénquima axial ausente o extremadamente raro en Laetia procera y Sloanea laurifolia; difuso y difuso en agregados en Aspidosperma excelsum, vasicéntrico unilateral en Buchenavia amazonia y en bandas delgadas en $L i$ cania micrantha. También son distintivos los radios de dos tamaños distintos en Laetia procera y presencia de punteaduras ornadas en Buchenavia amazonia, tilosis en Licania micrantha, cristales en Aspidosperma excelsum, Laetia procera y Sloanea laurifolia y de sílice en Licania micrantha.
\end{abstract}

Palabras clave: Madera, Aspidosperma excelsum, Licania micrantha, Buchenavia amazonia, Laetia procera, Sloanea laurifolia.

\footnotetext{
${ }^{1}$ Ingeniero Forestal, consultor independiente, Perú.

${ }^{2}$ Facultad de Ciencias Forestales, Universidad Nacional Agraria La Molina, Av. La Molina s/n, La Molina, Lima, Perú.

* Autor de Correspondencia: zarelacerdanobregon@gmail.com
} 


\begin{abstract}
The purpose of this research was to describe the anatomy of the following species of wood: Aspidosperma excelsum Benth., Licania micrantha Miq., Buchenavia amazonia Alwan \& Stace, Laetia procera (Poepp.) Eichler and Sloanea laurifolia (Benth.) Benth. The first three species were collected from Madre de Dios and last two from Ucayali, Peru. The anatomical description of wood species was determined in accordance with IAWA (1989) and IBAMA (1991) and included the evaluation of quantitative and qualitative xylem, data that showed the presence of common characteristics in cross grain, diffuse porous wood, simple perforation plates, alternate intervessel pits and vessels with appendices. Some distinct characteristics of each species were: absent or extremely rare axial parenchyma in Laetia procera and Sloanea laurifolia, diffuse parenchyma and diffuse-inaggregates parenchyma in Aspidosperma excelsum, one-sided vasicentric parenchyma in Buchenavia amazonia and narrow banded parenchyma in Licania micrantha. Additional distinctive characteristics observed included rays of two distinct sizes for Laetia procera, the presence of vestured pits in Buchenavia amazonia, the presence of tyloses in Licania micrantha, crystals in Aspidosperma excelsum, Laetia procera and Sloanea laurifolia and silica in Licania micrantha.
\end{abstract}

Key words: Wood, Aspidosperma excelsum, Licania micrantha, Buchenavia amazonia, Laetia procera, Sloanea laurifolia.

\section{Introducción}

En los últimos años existe una fuerte presión humana en la reducción de la cobertura de los bosques tropicales en forma selectiva; por lo cual, es necesario que nuevas especies potenciales sean identificadas y estudiadas para incrementar el volumen de madera producida por hectárea. La extracción selectiva de solo algunas especies fomenta que se comercialice un grupo reducido de especies, ocasionando un uso no sostenible del recurso.

La Amazonia peruana cuenta con una alta diversidad de especies maderables por unidad de área, que podría representar un gran suministro de materia prima para las industrias forestales dedicadas a la transformación. Por tanto, la sustitución de especies tradicionales por nuevas especies mediante su incorporación a la industria ayudaría al incremento de metros cúbicos extraídos por hectárea, contribuyendo así a un aprovechamiento integral de los bosques y obteniendo una mayor rentabilidad en el negocio maderero (León y Espinosa 2001).

Cury (2001) señala que los bosques amazónicos albergan una gran diversidad de especies que a su vez se encuentran en peligro debido a la sobreexplotación de estos recursos; por ello la necesidad de inventariar y caracterizar la biodiversidad florística para definir sus mecanismos de clasificación, utilización sostenible, potencial económico y conservación.

León y Espinosa (2001) mencionan que una especie es considerada importante desde el punto de vista comercial según los factores que influyen en la estructura anatómica y propiedades físico mecánicas, secado, preservado, entre otros; pues permite predecir las tecnologías de procesamiento y transformación para obtener un producto final de calidad a un menor costo. Sin embargo, actualmente, muchas especies nativas de la amazonia son empleadas de manera empírica en las industrias de la madera sin realizarse estudios previos de las propiedades de la madera lo que conlleva a un aprovechamiento no apropiado del recurso.

Los estudios anatómicos comprenden el nivel macroscópico que describe características que requieren poco o ningún aumento (características organolépticas y macroscópicas), y el nivel microscópico que describe las características de las células y tejidos que constitu- 
yen el leño de los árboles así como su organización, función y estructuras particulares que solo se diferencian con el uso del microscopio (Zenid y Ceccantini 2007).

La identificación de las maderas es importante tanto desde el punto de vista tecnológico como económico; además, es requerido en diferentes programas de fiscalización y regulación del comercio legal (Santini 2013). De esta manera se garantiza su desempeño de uso, en relación a su resistencia natural a organismos xilófagos o a las propiedades físico-mecánicas. El reconocimiento de las maderas es necesario también, como medida preventiva, en casos de sustitución de maderas de buena calidad por otras, aparentemente idénticas, pero de calidad inferior (De Lima 2011; Cury y Tomazello 2011).

El conocimiento anatómico de la madera a nivel de especie es fundamental en diferentes campos como arqueología terrestre y marina, bellas artes, restauración de edificios, comercio nacional e internacional y tráfico de especies, pues brinda información sobre los riesgos inherentes de la especie elegida y acerca de tratamientos preventivos contra agentes bióticos y abióticos; por ejemplo, en la restauración de elementos artísticos maderables proporciona información respecto al comportamiento de la madera al acabado, admisión de tintes y sustitución parcial de piezas (García et al. 2003).

El objetivo del presente trabajo fue caracterizar la estructura anatómica de cinco maderas provenientes de la selva amazónica peruana en las provincias de Tambopata (Departamento de Madre de Dios) y de Iparia (Departamento de Ucayali): Aspidosperma excelsum Benth., Licania micrantha Miq., Buchenavia amazonia Alwan \& Stace, Laetia procera (Poepp.) Eichler y Sloanea laurifolia (Benth.) Benth.

\section{Materiales y Métodos}

Las muestras de xiloteca fueron proporcionadas por el laboratorio de anatomía de la madera de la Universidad Nacional Agraria La Molina, las cuales provienen de la selva amazónica peruana en las provincias de Tambopata (Departamento de Madre de Dios) y de Iparia (Departamento de Ucayali), previamente fueron identificadas dendrológicamente (Cuadro 1). La preparación de las muestras se realizó en el Laboratorio de Anatomía de la Madera del Departamento Académico de Industrias Forestales, de la Facultad de Ciencias Forestales de la Universidad Nacional Agraria La Molina (Lima, Perú).

La descripción anatómica de la madera se realizó según las recomendaciones establecidas por IAWA (1989) e IBAMA (1991). Para la descripción de las características generales y macroscópicas se emplearon muestras de xiloteca de $2 \times 10 \times 15 \mathrm{~cm}$ debidamente orientadas y para la caracterización microscópica se prepararon láminas histológicas y de tejido macerado. Se calcularon parámetros estadísticos de los elementos anatómicos tales como: promedio, desviación estándar, coeficiente de variabilidad y valor máximo y mínimo de cada una de las especies que se indican en el Cuadro 4.

\begin{tabular}{|l|c|c|c|}
\hline \multicolumn{1}{|c|}{ Nombre Científico } & Nombre Común & Familia & Procedencia \\
\hline Aspidosperma excelsum Benth. & Remo caspi & Apocynaceae & Madre de Dios \\
\hline Licania micrantha Miq. & Apacharama & Chrysobalanaceae & Madre de Dios \\
\hline Buchenavia amazonia Alwan \& Stace & Nogal amarillo & Combretaceae & Ucayali \\
\hline Laetia procera (Poepp.) Eichler & Purma caspi & Salicaceae & Ucayali \\
\hline Sloanea laurifolia (Benth). Benth. & Huangana casha & Elaeocarpaceae & Ucayali \\
\hline
\end{tabular}

Cuadro 1. Especies para el estudio anatómico. 


\section{Resultados}

\section{Aspidosperma excelsum (Combretaceae)}

En condición seca al aire el duramen y la albura son de color amarillento. Anillos de crecimiento diferenciados por bandas oscuras irregulares. Olor y sabor no distintivos; grano entrecruzado, textura fina, brillo ausente, sin veteado. Poros: Visibles con lupa 10X, porosidad difusa, solitarios (Figura 1A), 56 poros/ $\mathrm{mm}^{2}$ clasificados como numerosos. Presencia de apéndices en uno o ambos lados de los elementos vasculares. Platinas de perforación simples. Parénquima: No visible con lupa $10 \mathrm{X}$, apotraqueal difuso y difuso en agregados (Figura 1A). Presencia de cristales de forma romboide (Figura 1B). Radios: Visibles con lupa 10X, en su mayoría biseriados, homocelulares, formados por células procumbentes (Figura $1 \mathrm{~B}$ y $1 \mathrm{C})$. Fibras: Libriformes de paredes muy gruesas.

\section{Licania micrantha (Chrysobalanaceae)}

En condición seca al aire el duramen es de color marrón amarillento, presenta transición gradual de albura a duramen. Anillos de crecimiento no diferenciados. Olor y sabor no distintivos. Grano entrecruzado, textura fina, brillo ausente y veteado en arcos superpuestos causado por el parénquima longitudinal. Poros: Visibles con lupa 10X, porosidad difusa, solitarios (Figura 2A), ocho poros $/ \mathrm{mm}^{2}$, clasificados como pocos. Presencia de apéndices en uno o ambos extremos de los elementos vasculares. Platinas de perforación simples. Presencia de tilosis (Figura 2A y 2C). Parénquima: Visible con lupa 10X, en bandas delgadas con una célula de ancho (Figura 2A). Radios: Visibles con lupa 10X, uniseriados, homocelulares, formados por células erectas o cuadradas. Presencia de sílice (Figura 2B y 2C). Fibras: Libriformes de paredes muy gruesas.

\section{Buchenavia amazonia (Combretaceae)}

En condición seca al aire existe transición gradual de albura a duramen; albura color amarillo pálido y el duramen amarillo anaranjado. Anillos de crecimiento diferenciados por bandas oscuras irregulares. Olor y sabor no distintivos. Grano entrecruzado, textura fina, brillo medio y veteado en arcos superpuestos causados por los anillos de crecimiento. Poros: Visibles con lupa 10X, porosidad difusa, predominantemente múltiples radiales (Figura $3 \mathrm{~A}), 35$ poros $/ \mathrm{mm}^{2}$, clasificados como moderadamente numerosos. Presencia de apéndices en uno o ambos lados de los elementos vasculares. Punteaduras ornadas. Platinas de perforación simples. Presencia de gomas (Figura 3A, 3C). Parénquima: No visible con lupa 10X, paratraqueal vasicéntrico unilateral escaso (Figura 3A). Radios: Visibles con lupa 10X, mayormente biseriados, homocelulares, formados por células erectas o cuadradas, y ocasionalmente heterocelulares formados por células procumbentes y una hilera marginal de células erectas (Figura 3B, 3C). Fibras: Septadas y libriformes de paredes delgadas.

\section{Laetia procera (Salicaceae)}

En condición verde y seca sin transición entre albura y duramen. De color amarillo pálido. Anillos de crecimiento diferenciados por bandas claras regulares. Olor y sabor no distintivos; grano entrecruzado, textura media, brillo medio, sin veteado. Poros: Visibles a simple vista, porosidad difusa, mayormente múltiples radiales (Figura $4 \mathrm{~A}$ ), nueve poros $/ \mathrm{mm}^{2}$, clasificados como pocos. Presencia de apéndices en un extremo de los elementos vasculares. Platinas de perforación simples. Parénquima: No visible con lupa de 10X, ausente o extremadamente raro (Figura 4A). Radios: Visibles con lupa de 10X, de dos tamaños distintos, predominantemente multiseriados, homocelulares, formados por células erectas o cuadradas, y heterocelulares, formados por células procumbentes y más de cuatro líneas marginales de células erectas o cuadradas. Presencia de radios longitudinalmente fusionados. Escasos cristales de forma cuadrada y romboide en células erectas y procumbentes (Figura $4 \mathrm{~B}$ y $4 \mathrm{C}$ ). Fibras: Septadas y libriformes de paredes gruesas.

\section{Sloanea laurifolia (Elaeocarpaceae)}

En condición seca existe transición gradual entre albura y duramen. La albura es de color marrón claro y el duramen de color marrón os- 

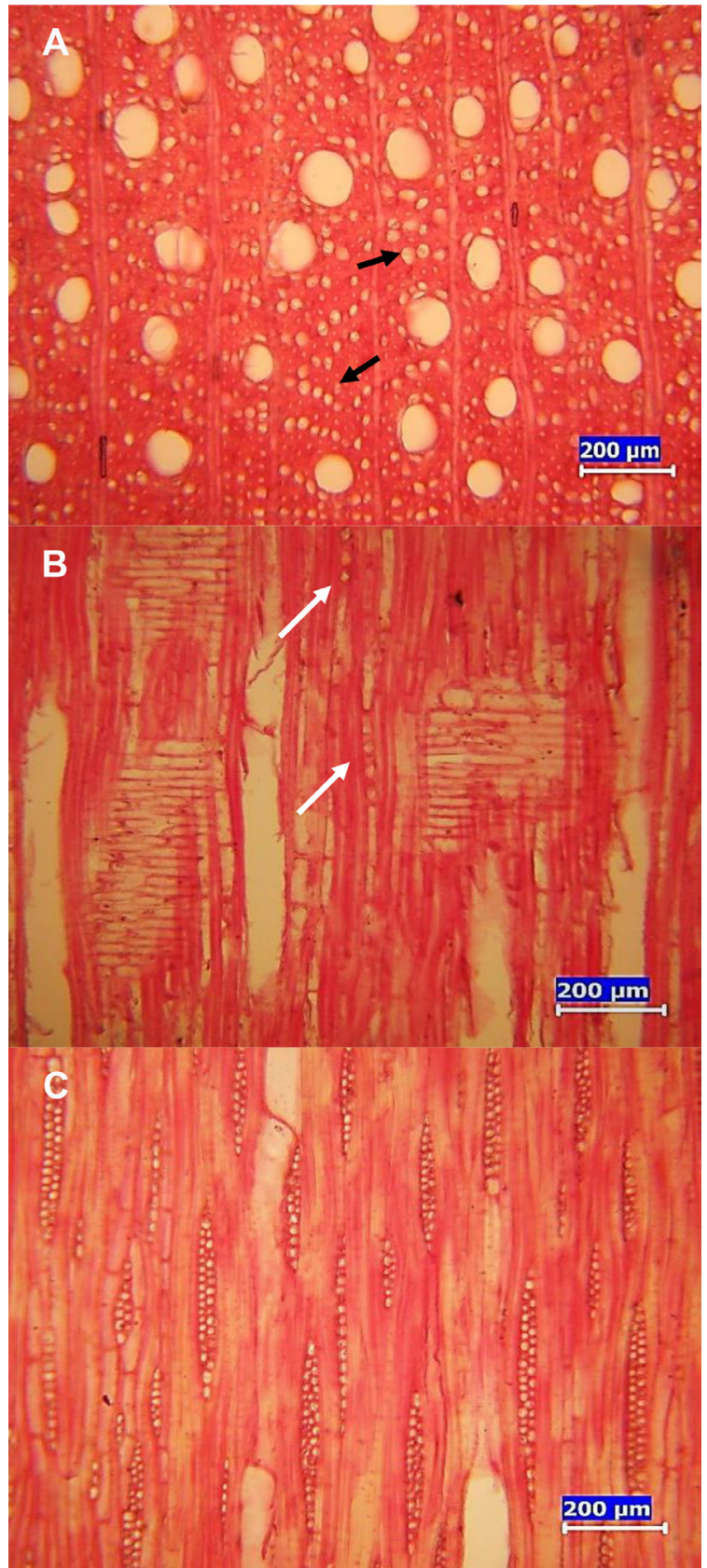

Figura 1. Caracteres anatómicos de la madera de Aspidosperma excelsum en vistas transversales, radiales y tangenciales. A: Porosidad difusa y poros solitarios; parénquima apotraqueal difuso y difuso en agregados (flechas negras). B y C: Radios predominantemente biseriados, homocelulares con células procumbentes. En B se muestran cristales de forma romboide en parénquima longitudinal (flechas blancas). 


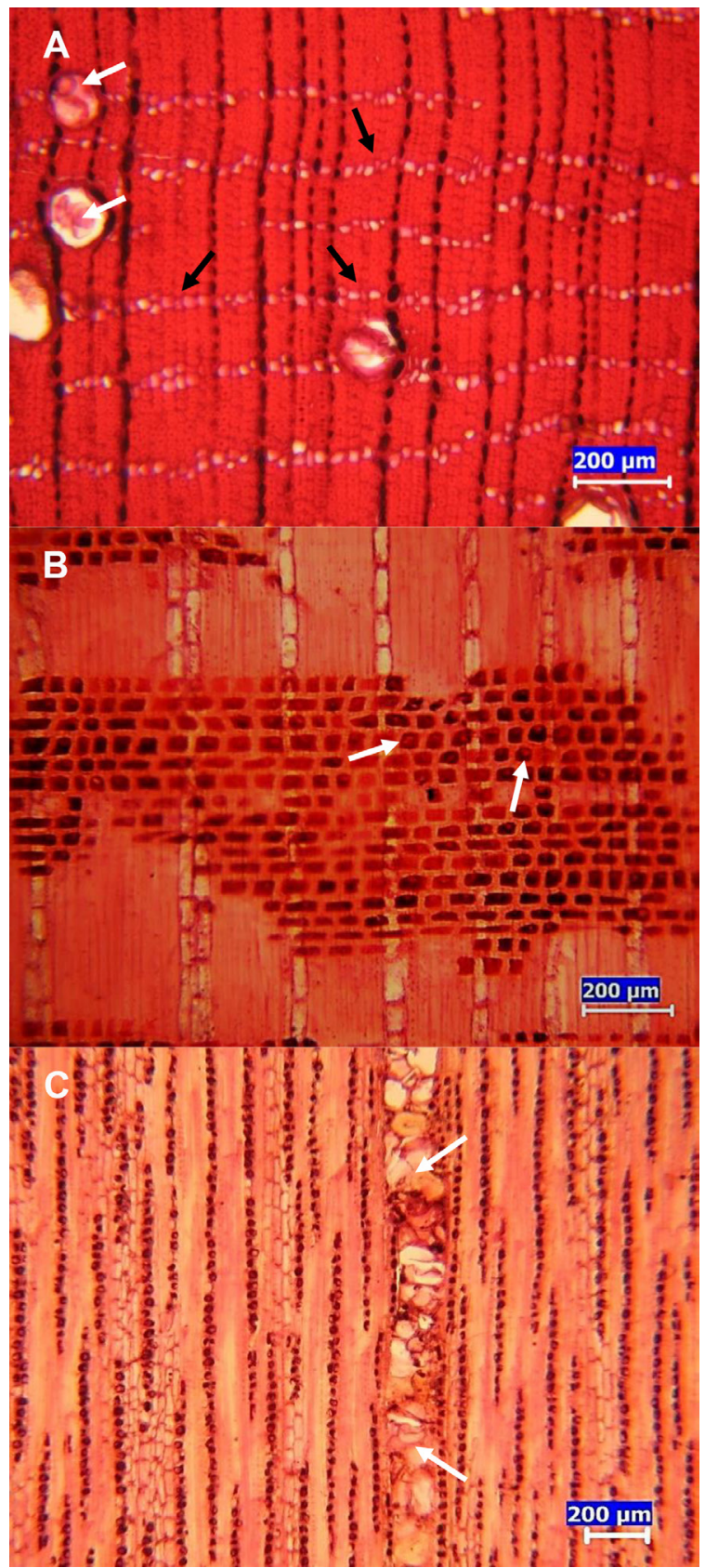

Figura 2. Caracteres anatómicos de la madera de Licania micrantha en vistas transversales, radiales y tangenciales. A: Porosidad difusa y poros solitarios. Parénquima en bandas delgadas con una célula de ancho (flechas negras). B y C: Radios uniseriados, homocelulares con células erectas o cuadradas, algunas conteniendo sílice (flechas blancas en B). En A y C las flechas blancas señalan tilosis. 

Madre de Dios y Ucayali, Perú

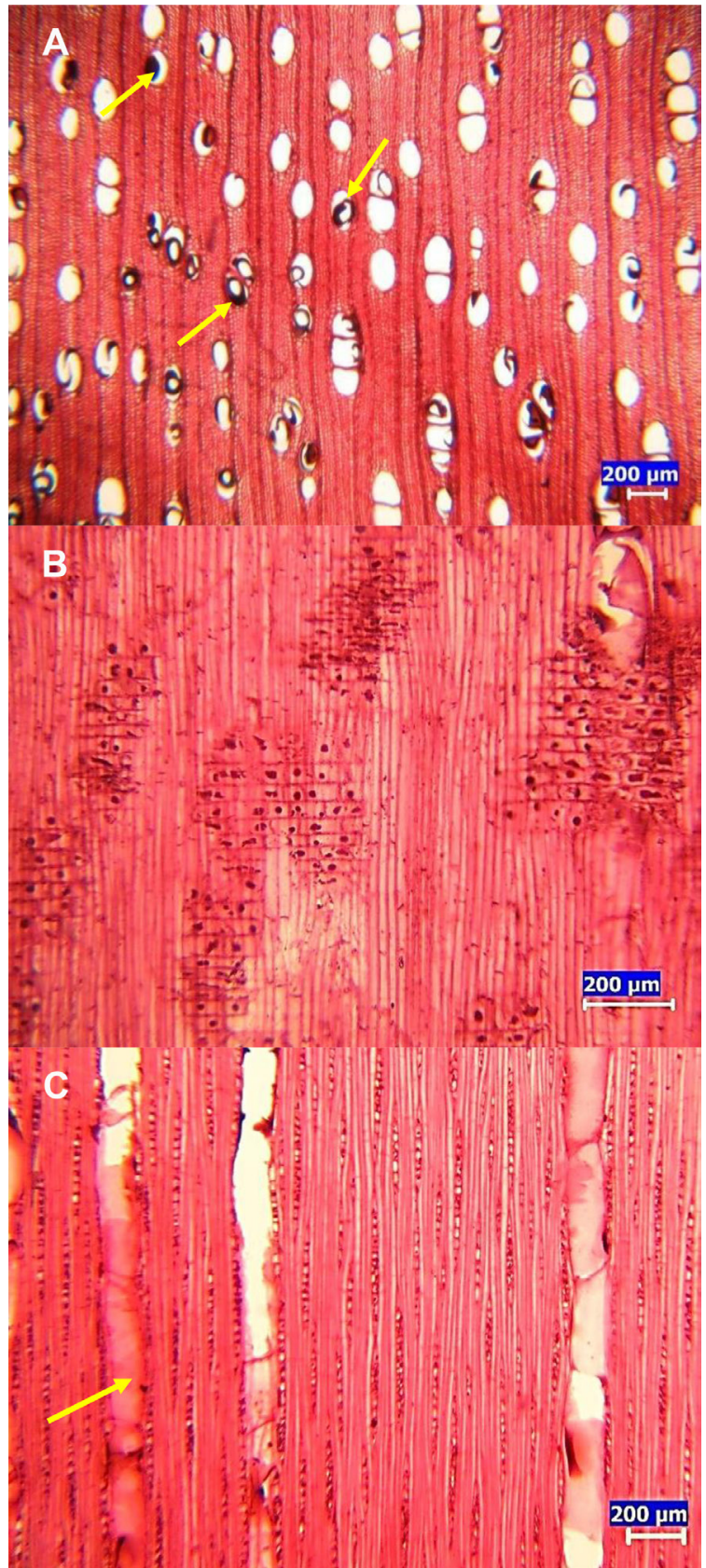

Figura 3. Caracteres anatómicos de la madera de Buchenavia amazonia en vistas transversales, radiales y tangenciales. A: Porosidad difusa y poros predominante múltiples radiales, parénquima paratraqueal vasicéntrico unilateral escaso y presencia de gomas (flechas amarillas). B y C: Radios biseriados, homocelulares y heterocelulares. 


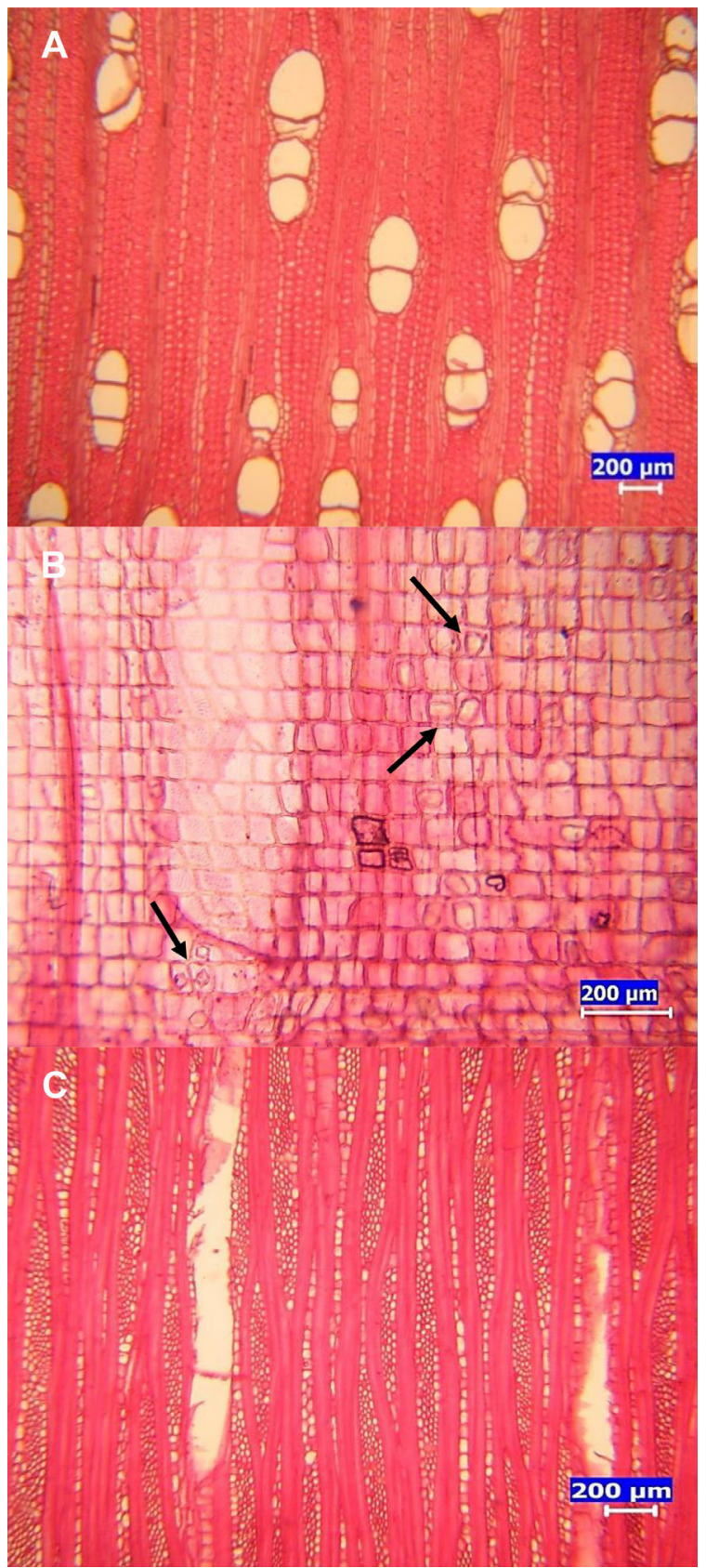

Figura 4. Caracteres anatómicos de la madera de Laetia procera en vistas transversales, radiales y tangenciales. A: Porosidad difusa y poros múltiples radiales, parénquima ausente o extremadamente raro. B y C: Radios de dos tamaños distintos, multiseriados, homocelulares (solo células erectas) y heterocelulares (células procumbentes y más de 4 líneas de células erectas). Radios longitudinalmente fusionados. En B se observa sílice en forma cuadrada y romboide en algunas células radiales (flechas negras). 


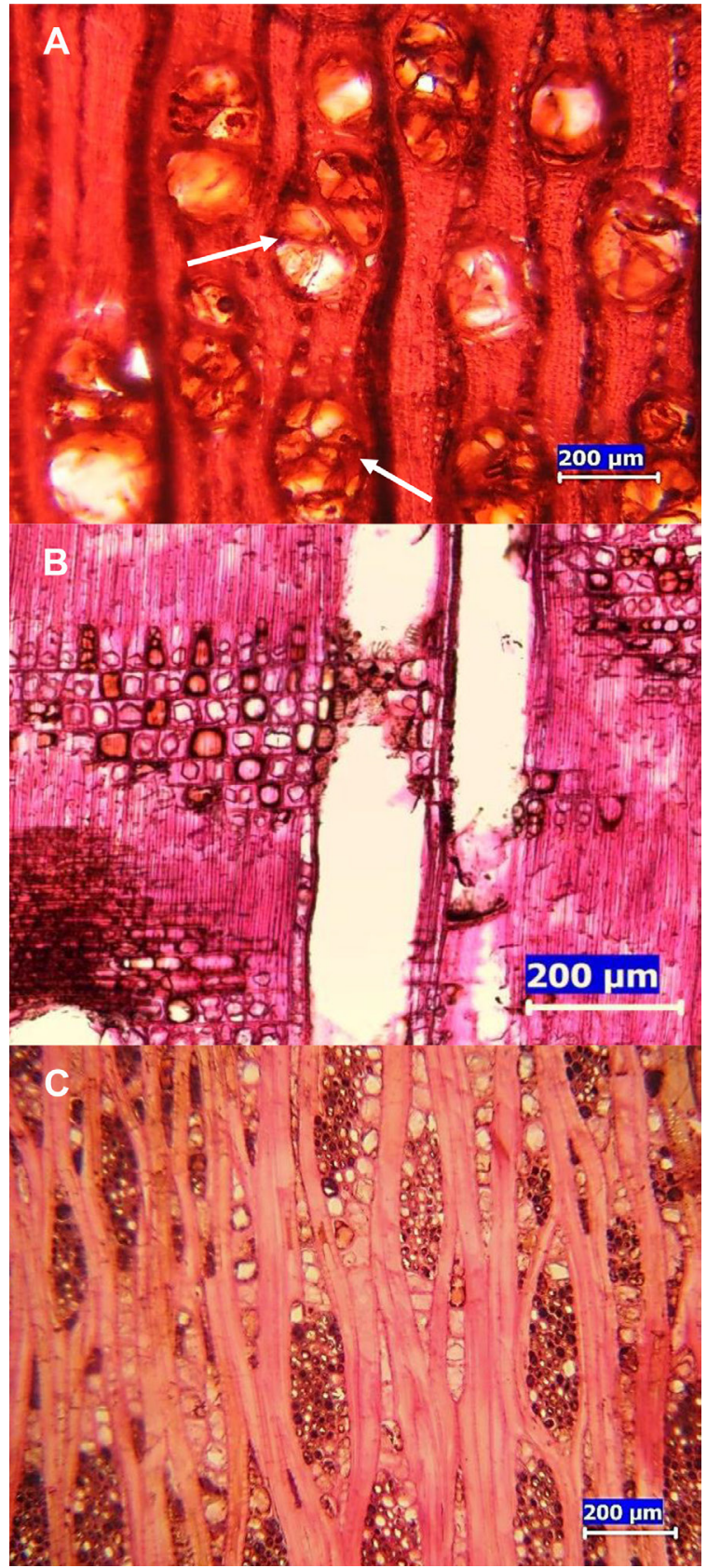

Figura 5. Caracteres anatómicos de la madera de Sloanea laurifolia en vistas transversales, radiales y tangenciales. A: Porosidad difusa y poros solitarios, parénquima ausente o extremadamente raro. B y C: Radios multiseriados, homocelulares (solo células erectas) y heterocelulares (células procumbentes y con más de 4 líneas de células erectas), con presencia de cristales. Las gomas se señalan con flechas en A. 
curo. Anillos de crecimiento no diferenciados. Olor y sabor no distintivos; grano entrecruzado, textura media, brillo ausente, no presenta veteado. Decoloraciones por oxidación. Poros: Visibles a simple vista, porosidad difusa, solitarios (Figura $5 \mathrm{~A}$ ), 18 poros $/ \mathrm{mm}^{2}$, clasificados como pocos. Presencia de apéndices en uno o ambos extremos de los elementos vasculares. Platinas de perforación simples. Presencia de gomas (Figura 5A). Parénquima: No visible con lupa de 10X, ausente o extremadamente raro (Figura 5A). Radios: Visibles con lupa $10 \mathrm{X}$, predominantemente multiseriados, homocelulares, formados por células erectas o cuadradas, y heterocelulares, formados por células procumbentes y más de cuatro líneas marginales de células erectas. Presencia de escasos cristales de forma cuadrada y romboide en células erectas y procumbentes, tres cristales por cámara. Presencia de gomas (Figura 5B). Fibras: Libriformes de paredes gruesas.

En los Cuadros 2 y 3 se presenta un resumen de las características generales, macroscópicas y microscópicas de las cinco especies estudiadas.

\section{Discusión}

Con relación al tipo de grano se puede indicar que las cinco especies estudiadas presentan grano entrecruzado, que según Desch y Dinwoodiem (1996) señalan, es un defecto muy común en áreas de nudos y cuando su presencia es excesiva, reduce seriamente la resistencia, además de acentuar las dificultades en el mecanizado.

En cuanto a textura, las especies Aspidosperma excelsum, Licania micrantha y Buchenavia amazonia presentan textura fina; mientras que Laetia procera y Sloanea laurifolia tienen textura media, condiciones que ya han sido reportadas por González (s/f), Miller y Détienne (2001) y Souza (2015).

Respecto al veteado, las especies Licania micrantha y Buchenavia amazonia presentan veteado en arcos superpuestos causados por el parénquima longitudinal y anillos de crecimiento, respectivamente; las otras especies no presentan veteado. González (s/f) coincide con lo señalado para Buchenavia amazonia.

A nivel macroscópico las especies que presentan poros predominantemente solitarios son Aspidosperma excelsum, Licania micrantha y Sloanea laurifolia; en tanto que Buchenavia amazonia y Laetia procera presentan poros predominantemente múltiples radiales. Détienne y Jacket (1983), Miller y Détienne (2001) y Souza (2015) coinciden con lo mencionado anteriormente; mientras que, González (s/f) difiere para Buchenavia amazonia.

El parénquima no es visible aún con lupa de 10X en Aspidosperma excelsum, Buchenavia amazonia, Laetia procera y Sloanea laurifolia; siendo visible con lupa 10X en Licania micrantha del tipo bandas angostas, resultados que coinciden con lo reportado por Détienne y Jacket (1983), Miller y Détienne (2001) y Souza (2015). Las especies Buchenavia amazonia y Sloanea laurifolia presentan inclusiones gomosas. Así mismo, Licania micrantha presenta tilosis, lo que coincide con lo señalado por Détienne y Jacket (1983), González (s/f) y León y Espinoza (2001).

A nivel microscópico, los elementos vasculares en Buchenavia amazonia son cortos; en Laetia procera son largos y en las otras tres especies son de longitud mediana. Las cinco especies estudiadas presentan platinas de perforación simples, punteaduras intervasculares alternas, mientras que la especie Buchenavia amazonia presenta punteaduras ornadas lo cual es señalado también por González (s/f). Igualmente, todas las especies presentan apéndices en los elementos vasculares en uno o ambos extremos y solo la especie Laetia procera presenta apéndice en un extremo. Wheeler et al. (2007) señalan que platinas de perforación simples y punteaduras intervasculares alternas son características comunes en maderas duras y en climas tropicales, la alta incidencia de esta característica refleja ventajas hidráulicas sobre platinas de perforación escalariformes y punteaduras opuestas. 


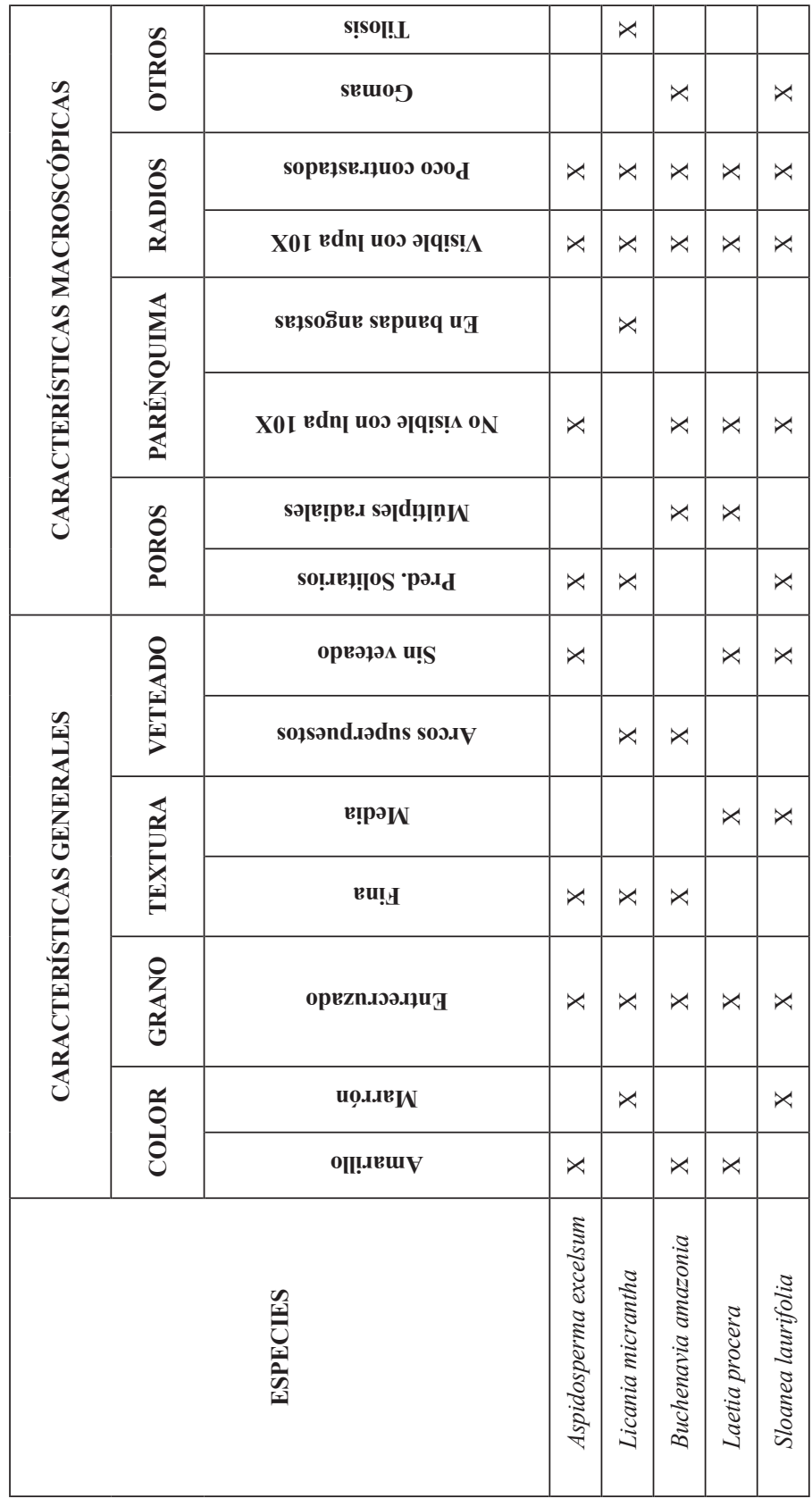




\begin{tabular}{|c|c|c|c|c|c|c|c|}
\hline \multirow{3}{*}{ 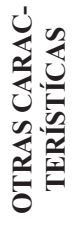 } & \multicolumn{2}{|c|}{ 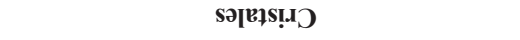 } & $x$ & & & $x$ & $x$ \\
\hline & \multicolumn{2}{|c|}{ sewoפ } & & & $x$ & & $x$ \\
\hline & \multicolumn{2}{|c|}{ әอ!!LS K S!SO!!L } & & $x$ & & & \\
\hline \multirow{6}{*}{$\frac{\sum^{n}}{\frac{1}{x}}$} & \multicolumn{2}{|c|}{ sepp̨dəS } & & & $x$ & $x$ & \\
\hline & \multirow{3}{*}{ 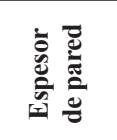 } & Esən.sô Kn్ & $x$ & $x$ & & & \\
\hline & & Esən.IP & & & & $x$ & $x$ \\
\hline & & врвоి|Ра & & & $x$ & & \\
\hline & \multirow{2}{*}{ pn!̣อินoT } & sหô.JeT & & & & $x$ & $x$ \\
\hline & & seur!̣p. & $x$ & $x$ & $x$ & & \\
\hline \multirow{5}{*}{$\frac{\tilde{0}}{\tilde{a}}$} & \multicolumn{2}{|c|}{ 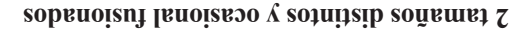 } & & & & $x$ & \\
\hline & \multicolumn{2}{|c|}{ 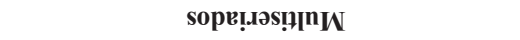 } & & & & $x$ & $x$ \\
\hline & \multicolumn{2}{|c|}{ 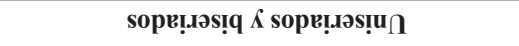 } & $x$ & $x$ & $x$ & $x$ & $x$ \\
\hline & \multicolumn{2}{|c|}{ 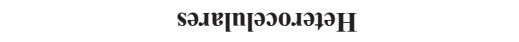 } & & & $x$ & $x$ & $x$ \\
\hline & \multicolumn{2}{|c|}{ sә.ле[п्әэошон } & $x$ & $x$ & $x$ & $x$ & $x$ \\
\hline \multirow{4}{*}{ 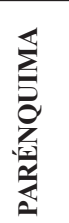 } & \multicolumn{2}{|c|}{ sełsoôur sepurg } & & $x$ & & & \\
\hline & \multicolumn{2}{|c|}{ 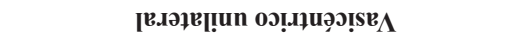 } & & & $x$ & & \\
\hline & \multicolumn{2}{|c|}{ 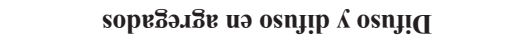 } & $x$ & & & & \\
\hline & \multicolumn{2}{|c|}{ 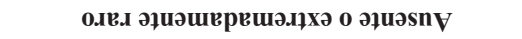 } & & & & $x$ & $x$ \\
\hline \multirow{8}{*}{ 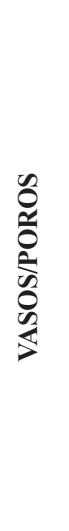 } & \multicolumn{2}{|c|}{ 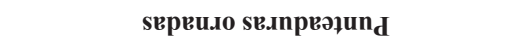 } & & & $x$ & & \\
\hline & \multicolumn{2}{|c|}{ 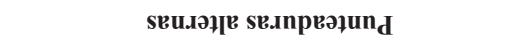 } & $x$ & $x$ & $x$ & $x$ & $x$ \\
\hline & \multirow{2}{*}{ 离 } & sour!pəW & & & & $x$ & $x$ \\
\hline & & souحənbəd & $x$ & $x$ & $x$ & & \\
\hline & \multicolumn{2}{|c|}{ 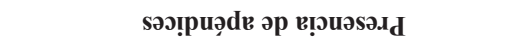 } & $x$ & $x$ & $x$ & $x$ & $x$ \\
\hline & \multirow{3}{*}{ 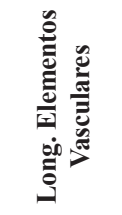 } & soถి.IBT & & & & $x$ & \\
\hline & & sоuє!рәш & $x$ & $x$ & & & $x$ \\
\hline & & soł.10 & & & $x$ & & \\
\hline & \multicolumn{2}{|c|}{ 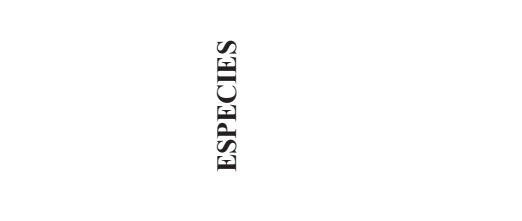 } & 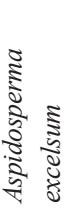 & 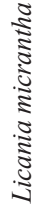 & 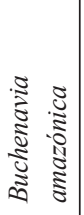 & 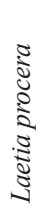 & 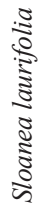 \\
\hline
\end{tabular}


Respecto al parénquima, en Laetia procera y Sloanea laurifolia el parénquima es ausente o extremadamente raro coincidiendo con lo afirmado por Miller y Détienne (2001) para la primera especie, mientras que para la segunda no coincide con lo descrito por Detienne y Jacquet (1983). En Aspidosperma excelsum es apotraqueal difuso y difuso en agregados; en Licania micrantha es en bandas angostas y en Buchenavia amazonia es paratraqueal vasicéntrico unilateral escaso, lo que concuerda con lo reportado por Souza (2015) y Detienne y Jacquet (1983). Carlquist (1988) menciona que la ausencia de parénquima representa ser una característica más primitiva en comparación con casos donde tienden a ser visibles. Estas células parenquimáticas se caracterizan por poseer paredes delgadas por lo que un aumento en la proporción de este tejido produce un efecto de reducción de la densidad (León 2010).

Aspidosperma excelsum y Licania micrantha son las únicas cuyos radios son exclusivamente homocelulares conformados por células procumbentes y erectas; respectivamente, resultados que confirma Souza (2015) para la primera especie, mientras que para la segunda no concuerda con lo reportado por Detienne y Jacquet (1983) lo que podría deberse a la genética de la especie y factores ambientales (Morris et al. 2015). Las otras tres especies presentan radios homocelulares y heterocelulares, lo cual fue observado igualmente por González (s/f), Miller y Détienne (2001) y Detienne y Jacquet (1983); destaca Laetia procera por presentar radios de tamaños distintos con presencia de algunos radios fusionados, resultados que coinciden con lo descrito en Insidewood (2004).

En cuanto a longitud de fibra, las especies Laetia procera y Sloanea laurifolia son de fibra larga, mientras que en las otras tres especies la longitud es mediana. Asimismo, el espesor de pared en Buchenavia amazonia es delgada, en Laetia procera y Sloanea laurifolia es gruesa y en Aspidosperma excelsum y Licania micrantha muy gruesa. Conocer el espesor de pared de las fibras permite predecir la densidad de la madera, es decir, se presenta un incremento en los valores de densidad a medida que aumenta el grosor de paredes de las fibras (Ziemińska et al. 2015; Ishiguri et al. 2009).

Las especies Buchenavia amazonia y Laetia procera presentan parénquima paratraqueal vasicéntrico escaso y parénquima raro o no frecuente, respectivamente. Además, ambas especies tienen fibras septadas. Wheeler et al. (2007) señalan que las maderas que poseen fibras septadas presentan raramente parénquima axial o está ausente o es escaso paratraqueal vasicéntrico en lugar de formas más elaboradas lo cual concuerda para ambas especies en el presente estudio. Carlquist (1988), Zheng y Martínez (2013) y otros autores señalan que las fibras septadas cumplen doble función, almacenamiento y soporte mecánico.

Licania micrantha presenta tilosis coincidiendo con lo reportado por León y Espinoza (2001); especies que poseen esta inclusión son destinadas preferentemente para la manufactura de barriles porque obstruye los vasos; además, la presencia de esta característica influye en el tiempo de secado, preservado, estabilidad dimensional, durabilidad natural y resistencia de la madera (Bowyer et al. 2003; Rodríguez et al. 2015).

Las especies Buchenavia amazonia y Sloanea laurifolia presentan gomas, cuya presencia en gran cantidad reducen la permeabilidad (Wangaard 1979 y Ninin 1985, citados por León y Espinoza 2001); además, de ocasionar efectos negativos en el proceso de aserrado debido a que interfiere con la evacuación del aserrín.

Finalmente, en Licania micrantha hay la presencia de abundante sílice, lo cual fue observado igualmente por Detienne y Jacquet (1983). La presencia de sílice en la madera produce desgaste rápido del filo cortante en las máquinas de aserrado (Roig 2011). Aspidosperma excel-

Cuadro 4. Valores estadísticos de los elementos xilemáticos para las cinco especies. ${ }^{\star}$ Radios pequeños, ${ }^{\star *}$ Radios grandes. (Cuadro en siguiente pagína). 


\begin{tabular}{|c|c|c|c|c|c|c|c|c|c|}
\hline \multirow[b]{2}{*}{ ESPECIES } & \multicolumn{3}{|c|}{ POROS } & \multicolumn{3}{|c|}{ RADIOS } & \multicolumn{3}{|c|}{ FIBRAS } \\
\hline & $\begin{array}{c}\varnothing \\
\text { Tangencial } \\
(\mu \mathrm{m})\end{array}$ & $\begin{array}{l}\mathbf{N} \% \\
\mathbf{m m}^{2}\end{array}$ & $\begin{array}{c}\text { Long. } \\
\text { elementos } \\
\text { vasculares } \\
(\mu \mathrm{m})\end{array}$ & $\begin{array}{c}\text { Altura } \\
(\mu \mathrm{m})\end{array}$ & $\begin{array}{c}\text { Ancho } \\
(\mu \mathrm{m})\end{array}$ & $\begin{array}{l}\mathrm{N} \% \\
\mathrm{~mm}\end{array}$ & $\begin{array}{c}\varnothing \text { Total } \\
(\mu \mathrm{m})\end{array}$ & $\begin{array}{c}\text { Espesor } \\
\text { Pared } \\
(\mu \mathrm{m})\end{array}$ & $\begin{array}{c}\text { Long. } \\
(\mu \mathrm{m})\end{array}$ \\
\hline \multicolumn{10}{|c|}{ Aspidosperma excelsum } \\
\hline Valor promedio & 60 & 56 & 494 & 283 & 23 & 6 & 19 & 7,0 & 1520 \\
\hline Valor mínimo & 40 & 23 & 244 & 177 & 17 & 4 & 14 & 4,3 & 1206 \\
\hline Valor máximo & 82 & 84 & 734 & 441 & 30 & 10 & 27 & 10,5 & 1869 \\
\hline Desv. Estándar & 9 & 12 & 104 & 53 & 3 & 1 & 3 & 1,2 & 158 \\
\hline $\mathrm{CV}(\%)$ & 15 & 21 & 21 & 19 & 12 & 20 & 14 & 17,6 & 10 \\
\hline \multicolumn{10}{|c|}{ Licania micrantha } \\
\hline Valor promedio & 67 & 8 & 606 & 325 & 21 & 13 & 20 & 7,9 & 1450 \\
\hline Valor mínimo & 40 & 4 & 312 & 119 & 15 & 9 & 13 & 5,2 & 920 \\
\hline Valor máximo & 93 & 14 & 959 & 629 & 32 & 17 & 29 & 11,1 & 1868 \\
\hline Desv. Estándar & 13 & 2 & 142 & 96 & 3 & 1 & 3 & 1,4 & 167 \\
\hline CV $(\%)$ & 20 & 26 & 23 & 30 & 14 & 11 & 17 & 17,8 & 12 \\
\hline \multicolumn{10}{|c|}{ Buchenavia amazonia } \\
\hline Valor promedio & 67 & 35 & 287 & 288 & 17 & 11 & 17 & 3,0 & 1114 \\
\hline Valor mínimo & 43 & 15 & 102 & 154 & 10 & 8 & 12 & 1,9 & 806 \\
\hline Valor máximo & 92 & 53 & 481 & 466 & 25 & 15 & 24 & 4,8 & 1367 \\
\hline Desv. Estándar & 10 & 11 & 89 & 61 & 3 & 2 & 3 & 0,6 & 135 \\
\hline $\mathrm{CV}(\%)$ & 15 & 31 & 31 & 21 & 19 & 14 & 15 & 18,5 & 12 \\
\hline \multicolumn{10}{|c|}{ Laetia procera } \\
\hline \multirow{2}{*}{ Valor promedio } & \multirow{2}{*}{137} & \multirow{2}{*}{9} & \multirow{2}{*}{821} & $526^{*}$ & 27 & \multirow{2}{*}{9} & \multirow{2}{*}{29} & \multirow{2}{*}{7,6} & \multirow{2}{*}{2358} \\
\hline & & & & $1678^{* *}$ & 64 & & & & \\
\hline \multirow{2}{*}{ Valor mínimo } & \multirow{2}{*}{93} & \multirow{2}{*}{5} & \multirow{2}{*}{437} & 241 & 8 & \multirow{2}{*}{6} & \multirow{2}{*}{17} & \multirow{2}{*}{4,7} & \multirow{2}{*}{2002} \\
\hline & & & & 834 & 39 & & & & \\
\hline \multirow{2}{*}{ Valor máximo } & \multirow{2}{*}{181} & & & 966 & 69 & & & & \\
\hline & & $1 J$ & (J) & 2682 & 112 & 12 & 41 & 10,0 & 2093 \\
\hline & & & & 160 & 18 & & & & \\
\hline Desv. Estantual & 17 & & 210 & 482 & 12 & 1 & 0 & 1,2 & 107 \\
\hline CY $(0 / 1$ & 14 & 17 & 27 & 30 & 66 & & 10 & 150 & \\
\hline$C v(\%)$ & 14 & 17 & 21 & 29 & 20 & 15 & 19 & 15,3 & 1 \\
\hline & & & loanea laurif & lia (Bentl & Benth & & & & \\
\hline Valor promedio & 111 & 18 & 585 & 530 & 65 & 10 & 20 & 5,0 & 1753 \\
\hline Valor mínimo & 76 & 10 & 280 & 239 & 35 & 7 & 14 & 3,0 & 1417 \\
\hline Valor máximo & 159 & 28 & 820 & 942 & 90 & 14 & 26 & 8,4 & 2176 \\
\hline Desv. Estándar & 17 & 3 & 124 & 141 & 11 & 2 & 3 & 0,9 & 157 \\
\hline CV (\%) & 15 & 17 & 21 & 27 & 18 & 15 & 14 & 19,1 & 9 \\
\hline
\end{tabular}


sum, Laetia procera y Sloanea laurifolia presentan cristales. Según Rodríguez et al. (2015) la formación de cristales en la madera también incide en el desgaste de las herramientas de trabajo cuando su presencia es abundante.

En el Cuadro 4 se presenta un resumen de los valores estadísticos de las dimensiones de los elementos xilemáticos para las cinco especies. De acuerdo a los coeficientes de variabilidad dados por Rubio (2002), se puede afirmar que el diámetro tangencial de poros en Laetia procera presenta datos $(10<\mathrm{CV} \%<15)$ regularmente homogéneos; mientras que las otras cuatro especies presentan datos regularmente variables a variables. Los datos de longitud de los elementos vasculares (CV\% > 25) en Buchenavia amazonia y Laetia procera son muy variables; en tanto que los datos de las otras tres especies $(20<\mathrm{CV} \%<25)$ son variables. Respecto al número de poros $/ \mathrm{mm}^{2}$, las especies Buchenavia amazonia y Licania micrantha presentan datos $(\mathrm{CV} \%>25)$ muy variables; mientras que las otras especies presentan datos $(15<\mathrm{CV} \%$ $<25)$ regularmente variables a variables. León y Quintero (2007) sostienen que la proporción de vasos obstruidos por tilosis, el diámetro de los vasos y la frecuencia de poros $/ \mathrm{mm}^{2}$ pueden ejercer influencia en relación al secado, preservado y propiedades de resistencia de la madera.

En las cinco especies el coeficiente de variabilidad del espesor de pared y diámetro total de las fibras presenta datos regularmente variables a muy variables; en tanto, para longitud de fibras las especies Laetia procera (CV: 7\%) y Sloanea laurifolia (CV: 9\%) presentan valores muy homogéneos y las otras tres especies reportan valores regularmente homogéneos.

Los datos obtenidos muestran una gran variabilidad en los elementos anatómicos de las cinco especies estudiadas. León y Quintero (2007) afirman que la variación en las características de la madera producida es debido a una serie de factores que influyen en el crecimiento de árbol. Factores como grado de madurez del cambium, factores ambientales como precipitación y temperatura, tratamientos silviculturales y factores genéticos; generan variabilidad en la madera porque afecta en la actividad del cambium (Pashin y de Zeeuw,
1980). Así también, Wheeler y bass (1998) y Beery et al. (1982) señalan que la posición en el árbol del cual se extrajo la muestra influye también en la variabilidad de la madera.

\section{Conclusiones}

Las cinco especies presentan grano entrecruzado; Laetia procera y Sloanea laurifolia poseen textura media, en tanto las especies Aspidosperma excelsum, Licania micrantha y Buchenavia amazonia son de textura fina.

Las cinco especies se caracterizan por presentar platinas de perforación simples y punteaduras alternas, además la madera de Buchenavia amazonia presenta punteaduras intervasculares ornadas y apéndices en uno o ambos extremos de los elementos vasculares.

Las maderas de Laetia procera y Buchenavia amazonia presentan fibras septadas.

Las especies Laetia procera y Sloanea laurifolia se caracterizan por presentar parénquima ausente o extremadamente raro o infrecuente; en Aspidosperma excelsum es difuso y difuso en agregados, en Licania micrantha aparece en bandas delgadas y es paratraqueal vasicéntrico unilateral escaso en Buchenavia amazonia.

La especie Licania micrantha presenta tilosis y abundante sílice; Buchenavia amazonia y Sloanea laurifolia presentan inclusiones gomosas; se observaron cristales en Aspidosperma excelsum, Laetia procera y Sloanea laurifolia.

La presencia de grano entrecruzado, alta densidad y presencia de sílice en Aspidosperma excelsum y Licania micrantha, podría afectar el desgaste de las herramientas cortantes.

El porcentaje elevado de tejido fibroso y el engrosamiento de la pared de las fibras en las especies Aspidosperma excelsum y Licania micrantha, tiene relación directa con la dureza y densidad de la madera

\section{Agradecimientos}

A la Sra. Susana Albengrin por su apoyo en la realización de esta investigación. 


\section{Bibliografía}

Beery, W; Ifju, G; McLain, T. 1982. Quantitative wood anatomy-relating anatomy to transverse tensile strength (en línea). Wood and Fiber Science 15(4): 395-407. Consultado 04 feb. 2017. Disponible en https://wfs.swst.org/index. $\mathrm{php/wfs/article/view/1215.}$

Bowyer, J; Shmulsky, R; Haygreen, J. 2003. Forest Products and Wood Science. An introduction. 4 ed. Iowa, US. Wiley-Blackwell. 554 p.

Carlquist, S. 1988. Comparative Wood Anatomy: Systematic, Ecological, and Evolutionary Aspects of Dicotyledon Wood (en línea). New York, US. Springer-Verlag Berlin Heidelberg. Consultado 08 feb. 2017. Disponible en https://books.google.com.pe/ books?hl=en \&lr $=\& \mathrm{id}=\mathrm{oHz} 1 \mathrm{CAAAQBA}-$ I\&oi=fnd\&pg=PA $1 \& \mathrm{dq}=$ S. + Carlquist + Comparative + Wood + Anatomy $+(1988 \&$ ots $=q 6$ T1ucmJD\&sig =5mDAjvareWYIdFicFgo icomPss $\# \mathrm{v}=$ onepage $\& \mathrm{q}=$ =S. $\% 20$ Carlquist $\% 20$ Comparative\%20Wood \%20Anatomy $\% 20$ (1988\&f=false.

Cury, G. 2001. Descrição da estrutura anatômica do lenho e sua aplicação na identificação de espécies arbóreas do cerrado e da mata Atlântica do Estado de São Paulo (en línea). Piracicaba, BR. Consultado 27 nov. 2016. Disponible en http://www.teses.usp.br/teses/disponiveis/11/11150/tde-23102002-145402/ptbr.php.

Cury, G; Tomazello, M. 2011. Descrição anatômica de espécies de madeira utilizadas na construção civil (en línea) Floresta e Ambiente 18(3):227-236. Consultado 17 ago. 2016. Disponible en http://www.floram.org/files/v18n3/ v18n3a1.pdf.

De Lima, M. 2011. Laminário virtual - UM software para auxílio na identificação de madeiras. Universidade Federal do Paraná. Curitiba (en línea). Curitiba, BR. Consultado 07 ago. 2016. Disponible en http://www.madeira.ufpr. br/tccpublicados/tccmicheletelles.pdf.

Desch, H; Dinwoodiem, J. 1996. TIMBER Structure, Properties, Conversion and Use (en línea). 7 ed. s.l. Macmillan Education
UK. Consultado 25 ene. 2017. Disponible en https://link.springer.com/book/10.1007\% 2F978-1-349-13427-4.

Détienne, P; Jacket, P. 1983. Atlas d'identification des bois de Lamazonie et des régions voisines. Marne, FR. Centre Technique Forestier Tropical. $82 \mathrm{p}$.

García, L; Guindeo, A; Peraza, C; De Palacios, P. 2003. La madera y su tecnología: Anomalías y defectos, estructura microscópica de coníferas y frondosas, identificación de maderas, descripción de especies y pared celular. Madrid, ES . AITIM. 327 p.

González, I. s/f. Atlas de maderas Selva Central (en línea). s.l. Consultado 20 ago. 2016. Disponible en https://issuu.com/nicanorebook/ docs/atlas de maderas ebook.

IAWA (International Association of Wood Anatomists, NL). 1989. IAWA list of microscopic features for hardwood identification with an appendix on non-anatomical information. Leiden, NL. Boletín 10(3):19-332.

IBAMA (Instituto Brasileiro do Meio Ambiente e dos Recursos Naturais Renováveis, BR). 1991. Normas de procedimentos em estúdios de anatomia da madeira: I. Angiospermae, II. Gimnospermae. 19 p.

INSIDEWOOD (Inside Wood Database). 2004. (en línea). Consultado 10 sep. 2016 Disponible en http://insidewood.lib.ncsu.edu/search.

Ishiguri, F; Hiraiwa, T; Iizuka, K; Yokota, S; Priadi, D; Sumiasri, N; Yoshizawa, N. 2009. Radial variation of anatomical characteristics in Paraserianthes falcataria planted in Indonesia (en línea). IAWA Journal 30(3):343-352. Consultado 14 ene. 2017. Disponible en https://www. researchgate.net/publication/265625470 Radial Variation of Anatomical Characteristics in Paraserianthes Falcataria Planted in Indonesia.

León, W. 2010. Anatomía y densidad o peso específico de la madera (en línea). Revista Forestal Venezolana 54(1):67-76. Consultado 07 feb. 2017. Disponible en http://www.saber.ula. ve/bitstream/123456789/31646/1/ensayo1.pdf 
León, W; Espinoza, N. 2001. Anatomía de la Madera. Mérida, VE. Universidad de los Andes. $396 \mathrm{p}$.

León, W; Quintero, M. 2007. Variabilidad de los vasos en Gmelina arborea Roxb. (Verbenaceae) proveniente de plantaciones del estado Portuguesa (Venezuela) (en línea). Revista Forestal Venezolana 51(1):97-113. Consultado 11 feb. 2017. Disponible en http://www.saber.ula.ve/ bitstream/123456789/24467/2/articulo10.pdf.

Miller, R; Détienne, P. 2001. Major Timber Trees of Guyana. Wood Anatomy (en línea). Tropenbos International. Consultado 08 ago. 2015. Disponible en www.tropenbos.org/file.php/1625/ tbiseries-20.pdf.

Morris, H; Plavcová, L; Cvecko, P; Fichtler, E; Gillingham, M; Martínez, H; McGlinn, D; Wheeler, E; Zheng, J; Ziemińska, K; Jansen, S. 2015. A global analysis of parenchyma tissue fractions in secondary xylem of seed plants (en línea). New Phytologist 209(4):1553-1565. Consultado 16 ene. 2017. Disponible en http://onlinelibrary.wiley.com/doi/10.1111/nph.13737/ full.

Panshin, A; De Zeeuw, C. 1980. Textbook of wood technology. McGraw-Hill Series in Forest Resources. New York. 722.

Rodríguez, R; Ramírez, A; Palacios, H; Fuentes, F; Silva, J; Saucedo, A. 2015. Características anatómicas, físico-mecánicas y de maquinado de la madera de mezquite (Prosopis velutina Wooton) (en línea). Revista Mexicana de Ciencias Forestales. 6(28):156-173. Consultado 14 feb. 2017. Disponible en https://issuu.com/cienciasforestales/docs/vol.6 no.28.

Roig, F. 2011. MADERA Formación, composición y estructura anatómica (en línea). Universidad Nacional de Cuyo - CONICET. Consultado 14 sep. 2016. Disponible en http:// campus.fca.uncu.edu.ar/pluginfile.php/9447/ mod_resource/content/0/Madera_2011.pdf.

Rubio, J. 2002. Estadística. Universidad Nacional Agraria La Molina. Lima, PE. 175 p.

Santini, L. 2013. Descrição macroscópica e microscópica da madeira aplicada na identificação das principais espécies comercializadas no Estado de São Paulo - Programas "São Paulo da Amazônia" e "Cadmadira". Consultado 20 may. 2018. Disponible en http://www.teses.usp.br/ teses/disponiveis/11/11150/tde-11092013151731/pt-br.php.

Souza, A. 2015. Anatomia da madeira de quatro espécies de Aspidosperma Mart. \& Zucc. Comercializadas no estado do Pará, Brasil (en línea). Ciência da Madeira (Brazilian Journal of Wood science) 6(1):47-62. Consultado 25 ago. 2016. Disponible en https://periodicos.ufpel. edu.br/ojs2/index.php/cienciadamadeira/article/view/5444/cienciadamadeirav6n1a6.

Wheeler, E; Baas, P. 1998. Wood identification - A review (en línea). IAWA Journal 19(3):241-264. Consultado 20 may. 2018. Disponible en https:// www.researchgate.net/publication/270681002 Wood Identification -A Review.

Wheeler, E; Baas, P; Rodgers, S. 2007. Variations in dicot wood anatomy: A global analysis based on the Insidewood database (en línea). IAWA Journal 28(3):229-258. Consultado 21 mar. 2017. Disponible en https://www.researchgate. net/publication/239547088 Variations In Dieot Wood Anatomy A Global Analysis Based on the Insidewood Database.

Zenid, J; Ceccantini, G. 2007. Identificação macroscópica de madeiras. Instituto de Pesquisas Tecnológicas do Estado d São Paulo - IPT (en línea). Consultado 28 abr. 2016. Disponible en http://www.celso-foelkel.com.br/artigos/outros/ Apostila-Identifica\%E7\%E3o\%20de\%20madeiras.pdf.

Zheng, J; Martínez, H. 2013. Wood anatomical correlates with theoretical conductivity and wood density across China: evolutionary evidence of the functional differentiation of axial and radial parenchyma (en línea). Annals of Botany 112(5):927-935. Consultado 26 ene. 2017. Disponible en https://academic.oup.com/ aob/article/112/5/927/140030/Wood-anatomical-correlates-with-theoretical.

Ziemińska, K; Westoby, M; Wright, I. 2015. Broad Anatomical Variation within a Narrow Wood Density Range-A Study of Twig Wood 
across 69 Australian Angiosperms (en línea). Consultado 22 ene. 2017. Disponible en http:// journals.plos.org/plosone/article?id=10.1371/ journal.pone.0124892

Presentado: 24/01/2018

Aceptado: 08/06/2018 